\title{
OPTICAL KERR TYPE NONLINEARITY
}

\author{
J. ZALEŚNY \\ Institute of Physics, Technical University of Szczecin \\ Al. Piastów 48, 70-310 Szczecin, Poland
}

\begin{abstract}
The origin of the Kerr type nonlinearity of the medium as a result of the interaction between photons via the Dirac delta-potential is presented in the formalism adopted from the photon wave function approach. In the view of the result the optical soliton may be treated as a bound state (cluster) of many photons.
\end{abstract}

PACS numbers: $42.65 .-\mathrm{k}$

\section{Introduction}

To describe photon in terms of a one-particle wave function, i.e., on the first quantization level, we follow the way presented in [1-3]. Another approach was proposed in [4]. The concept of the position-representation photon wave function has a long history and is still controversial. Nevertheless, we do not want to discuss the question here. The reader interested in this problem is referred to [1-4] and references therein. In this paper the concept serves us as a convenient tool.

The starting point for us is the free photon Schrödinger equation [1-3]. On this basis we develop the description of photon in medium in terms of the photon wave function. Some attempts of this kind have been presented in [1-3]. What is new in our approach is to show that the influence of the medium on the photon can be described through some potentials. Though generally the idea is not new, see e.g. $[5,6]$, but here we realize it within the formalism of the photon wave function. This approach can be easily extended to the case of many interacting photons. For a large number of photons, it results as a nonlinear optics. In particular, taking as a simple model the photons interacting via the Dirac delta-potential one obtains (in the Hartree approximation) the Kerr type nonlinearity of the medium. The problem of interactions of photons via delta-potential and the Kerr nonlinearity of the medium has its vast literature, especially in the context of quantization of nonlinear Schrödinger equation $([7,8]$ and references therein). But all the literature refers to the procedure of creation-annihilation operators.

The paper is organized in the following way. We begin Sec. 2 with the brief description of a single photon in the medium. On this basis we extend the approach to the case of many interacting photons. We present that in Hartree approximation 
the many-photon quantum Schrödinger equation takes on the form similar to the nonlinear Schrödinger equation. The obtained equation expressed in terms of classical language is nothing else but the Maxwell equations with material relations in the form of the Kerr type nonlinearity. In Sec. 3 we summarize results and give some final remarks.

\section{Results}

In papers [1-3] the following form of the Schrödinger equation for free photon was proposed:

$$
\begin{aligned}
& \mathrm{i} \hbar \partial_{t} \mathrm{~F}=H_{\mathrm{f}} \mathrm{F}, \\
& \mathrm{F}=\left[\begin{array}{c}
\boldsymbol{E}(t, r)+\mathrm{i} \boldsymbol{H}(t, \boldsymbol{r}) \\
\boldsymbol{E}(t, \boldsymbol{r})-\mathrm{i} \boldsymbol{H}(t, \boldsymbol{r})
\end{array}\right], \quad H_{\mathrm{f}}=c\left[\begin{array}{cc}
\boldsymbol{p} \cdot \boldsymbol{S} & 0 \\
0 & -\boldsymbol{p} \cdot \boldsymbol{S}
\end{array}\right],
\end{aligned}
$$

$p=-\mathrm{i} \hbar \nabla-$ momentum of photon, $\left(\boldsymbol{S}_{i}\right)_{k l}=-\mathrm{i} \varepsilon_{i k l}-$ spin photon matrix. In the classical language, the equations are equivalent to the following Maxwell equations:

$$
\partial_{t} \boldsymbol{E}=c \nabla \times \boldsymbol{H}, \quad \partial_{t} \boldsymbol{H}=-c \nabla \times \boldsymbol{E}, \quad \boldsymbol{D}=\boldsymbol{E}, \quad \boldsymbol{B}=\boldsymbol{H} .
$$

describing free fields in vacuum. Since all the information carried by function $F$ is contained in its positive energy (positive frequency) part $F^{(+)}$, one may take this part as the true photon wave function [3]

$$
\psi=\mathrm{F}^{(+)} \text {. }
$$

To become a complete set of the Maxwell equations, Eq. (3) must be supplied by divergence conditions $\nabla \cdot \boldsymbol{E}=0, \nabla \cdot \boldsymbol{H}=0$. It is equivalent to the relation $\boldsymbol{p} \cdot \psi=0$.

In order to describe the propagation of photon in dielectric, one should include in Hamiltonian the interaction term. On the microscopic level, such interaction is rather complicated, but here we will take it into account in a phenomenological way. We assume that the photon treated as a quantum object "feels" the medium as an external classical field. For a stationary state the wave function takes on the form

$$
\psi_{\omega}=\varphi_{\omega}(\boldsymbol{r}) \exp (-\mathrm{i} \omega t), \quad \text { where } \quad \varphi_{\omega}(\boldsymbol{r})=\left[\begin{array}{c}
\boldsymbol{E}(\boldsymbol{r})+i \boldsymbol{H}(\boldsymbol{r}) \\
\boldsymbol{E}(\boldsymbol{r})-i \boldsymbol{H}(\boldsymbol{r})
\end{array}\right]
$$

Generally, the couplings of the medium with the electric and magnetic part of the wave function may be different. To take it into account we introduce two real and symmetric matrices $\gamma$ and $\eta$ which split the wave function $\psi_{w}$ into electric and magnetic parts

$$
\begin{aligned}
& \gamma \psi_{\omega}+\eta \psi_{\omega}=\psi_{\omega}, \\
& \gamma \psi_{\omega}=\left[\begin{array}{r}
\boldsymbol{E} \\
\boldsymbol{E}
\end{array}\right] \quad \text { and } \quad \eta \psi_{\omega}=\left[\begin{array}{c}
i \boldsymbol{H} \\
-i \boldsymbol{H}
\end{array}\right], \\
& \eta=\frac{1}{2}\left[\begin{array}{rr}
1 & -1 \\
-1 & 1
\end{array}\right] \quad \text { and } \gamma=\frac{1}{2}\left[\begin{array}{ll}
1 & 1 \\
1 & 1
\end{array}\right] .
\end{aligned}
$$


The projection operators $\gamma, \eta$ fulfill the following relations i.e.: $\gamma^{2}=\gamma, \eta^{2}=$ $\eta, \gamma \eta=0$.

When the couplings with the electrical and magnetic parts are taken into account, the Schrödinger equation takes on the form

$$
\hbar \omega \psi_{\omega}=H_{\mathrm{f}} \psi_{\omega}-\Omega_{\omega}(\boldsymbol{r}) \gamma \psi_{\omega}-\Gamma_{\omega}(\boldsymbol{r}) \eta \psi_{\omega} .
$$

$\Omega_{\omega}(\boldsymbol{r}) \gamma, \Gamma_{\omega}(\boldsymbol{r}) \eta$, have interpretation of potential energy operators. $\Omega_{\omega}(\boldsymbol{r})$ is connected with the dielectric susceptibility $\chi_{\omega}(r)=(1 / 4 \pi) \Omega_{\omega}(r) / \hbar \omega$, and $\Gamma_{\omega}(r)$ with magnetic susceptibility $\chi_{\omega}^{\mathrm{m}}(r)=(1 / 4 \pi) \Gamma_{\omega}(\boldsymbol{r}) / \hbar \omega$.

Let us now consider $N$ interacting photons propagating in a homogeneous medium. Let the Hamiltonian $H$ have the form

$$
H=\sum \mathrm{f} H_{\mathrm{f}}-N \Omega_{\omega 0} \gamma-\sum_{\mathrm{j}>\mathrm{f}} H_{\mathrm{fj}}
$$

where the first term is the sum of $N$ identical Hamiltonians $H_{\mathrm{f}}$ in the form given by Eq. (2) - each describing evolution of a free photon, the second term describes their coupling to the non-magnetic medium $\left(\Gamma_{\omega}=0\right)$, and the third term is the sum taken over all the photon-photon interactions $H_{\mathrm{fj}}$. Any mechanism can lead effectively to the weak interaction between photons. The main assumption is that the interaction can be modeled by the Dirac $\delta$-function (when the two photons are simultaneously in a very small volume element $V$ of the space). The measure of its intensity is given by a parameter $A_{\omega}$. Because the photon-photon interaction is performed with the help of the medium, and the dielectric medium acts effectively only on the electrical component of the wave function, therefore the matrix $\gamma$ should appear in the Hamiltonian $H_{\mathrm{fj}}$

$$
H_{\mathrm{fj}}=A_{\omega} V \gamma \delta\left(r_{\mathrm{f}}-r_{\mathrm{j}}\right) \text {. }
$$

The Schrödinger equation for the $N$ photons takes on the form

$$
\mathrm{i} \hbar \partial_{t} \psi\left(r_{1}, \ldots, r_{N}, t\right)=H \psi\left(r_{1}, \ldots, r_{N}, t\right) .
$$

The position-representation $N$-photon wave function $\psi\left(r_{1}, \ldots, r_{N}, t\right)$ is constructed in a similar way as the one constructed previously for one photon

$$
\psi=\left[\begin{array}{c}
\tilde{\boldsymbol{E}}\left(t, \boldsymbol{r}_{1}, \ldots, \boldsymbol{r}_{N}\right)+\mathrm{i} \tilde{\boldsymbol{H}}\left(t, \boldsymbol{r}_{1}, \ldots, \boldsymbol{r}_{N}\right) \\
\tilde{\boldsymbol{E}}\left(t, \boldsymbol{r}_{1}, \ldots, \boldsymbol{r}_{N}\right)-\mathrm{i} \tilde{\boldsymbol{H}}\left(t, \boldsymbol{r}_{1}, \ldots, \boldsymbol{r}_{N}\right)
\end{array}\right]=\phi_{\omega}\left(\boldsymbol{r}_{1}, \ldots, \boldsymbol{r}_{N}\right) \exp (-\mathrm{i} \omega t)
$$

The last equality means that only stationary states are considered. In the Hartree approximation, see e.g. [9], $\phi_{\omega}$ can be written as a product of one-photon wave functions $\varphi_{j}$

$$
\begin{aligned}
& \phi_{\omega}=\prod_{f=1}^{N} \varphi_{f} \\
& =\frac{1}{\sqrt{8 \pi \hbar \omega}}\left[\begin{array}{c}
e\left(\boldsymbol{r}_{1}\right)+i h\left(\boldsymbol{r}_{1}\right) \\
e\left(\boldsymbol{r}_{1}\right)-i h\left(\boldsymbol{r}_{1}\right)
\end{array}\right] \ldots \frac{1}{\sqrt{8 \pi \hbar \omega}}\left[\begin{array}{c}
\boldsymbol{e}\left(\boldsymbol{r}_{N}\right)+\mathrm{i} \boldsymbol{h}\left(\boldsymbol{r}_{N}\right) \\
\boldsymbol{e}\left(\boldsymbol{r}_{N}\right)-\mathrm{i} \boldsymbol{h}\left(\boldsymbol{r}_{N}\right)
\end{array}\right] \\
& =\left(\frac{1}{\sqrt{8 \pi \hbar \omega}}\right)^{N}\left[\begin{array}{c}
\left(e\left(\boldsymbol{r}_{1}\right)+\mathrm{i} \boldsymbol{h}\left(\boldsymbol{r}_{1}\right)\right) \ldots\left(\boldsymbol{e}\left(\boldsymbol{r}_{N}\right)+\mathrm{i} \boldsymbol{h}\left(\boldsymbol{r}_{N}\right)\right) \\
\left(e\left(\boldsymbol{r}_{1}\right)-\mathrm{i} h\left(\boldsymbol{r}_{1}\right)\right) \ldots\left(\boldsymbol{e}\left(\boldsymbol{r}_{N}\right)-\mathrm{i} \boldsymbol{h}\left(\boldsymbol{r}_{N}\right)\right)
\end{array}\right] .
\end{aligned}
$$


The three-dimensional vectors $\tilde{\boldsymbol{E}}$ and $\tilde{\boldsymbol{H}}$ by no means can be treated as a classical electric and magnetic field. In the case of one photon the vectors $\boldsymbol{e}$ and $\boldsymbol{h}$ can be interpreted as an electric and magnetic field connected with the photon. In general, our point of view is that in many-photon case, the vectors of an effective, self-consistent one-photon wave function could be interpreted as a classicalelectric and magnetic field.

Multiplying (12) by the product $\prod_{f \neq 1}^{N} \varphi_{f}^{+}$, taking integral over coordinates of the particles, i.e. $\int \mathrm{d} r_{2} \mathrm{~d} r_{3} \ldots \mathrm{d} r_{\mathrm{N}}$, and assuming the normalization of the one-particle function

$$
\int \varphi_{f}^{+} \varphi_{f} \mathrm{~d} r=1
$$

one obtains the one-particle self-consistent equation

$$
\hbar \omega \varphi=H_{\mathrm{f}} \varphi-\Omega_{\omega} \gamma \varphi-(N-1) A_{\omega} V\left(\varphi^{+} \varphi\right) \gamma \varphi,
$$

where the renormalized medium potential $\Omega_{\omega}$ is

$$
\Omega_{\omega}=\Omega_{\omega 0}+2(N-1) \frac{A_{\omega} V}{(8 \pi \hbar \omega)^{2}} \int\left(\boldsymbol{e}^{2}+h^{2}\right) \boldsymbol{e}^{2} \mathrm{~d} \boldsymbol{r} .
$$

The row vector $\varphi^{+}$means the Hermitian adjoint of $\varphi$, and the integrals are taken over all the space. The physical meaning of the constant $\Omega_{\omega}$ is similar as previously was $\Omega_{w 0}$, i.e., in case of nonlinear medium it is connected with the linear (background) part of the dielectric constant.

In the next step one may recast $\mathbf{E q}$. (16) to ordinary form of the Maxwell equations

$$
\frac{\omega}{c}\left[1+4 \pi \chi_{\omega}+\alpha_{\omega}(N-1)\left(e^{2}+h^{2}\right)\right] e=\mathrm{i} \nabla \times h, \quad \frac{\omega}{c} h=-\mathrm{i} \nabla \times e,
$$

where $\chi_{\omega}=\Omega_{\omega} / 4 \pi \hbar \omega$ and $\alpha_{\omega}=\frac{1}{4 \pi} \frac{A_{\omega} V}{(\hbar \omega)^{2}}$.

The quantities $\boldsymbol{e}$ and $h$ require some renormalization

$$
\boldsymbol{E}_{\mathrm{cl}}=\sqrt{N-1} \boldsymbol{e}, \quad \boldsymbol{H}_{\mathrm{cl}}=\sqrt{N-1} \boldsymbol{h} .
$$

Therefore Eq. (18) takes on the form

$$
\frac{\omega}{c}\left[\varepsilon_{\omega}+\alpha_{\omega}\left(\boldsymbol{E}_{\mathrm{cl}}^{2}+\boldsymbol{H}_{\mathrm{cl}}^{2}\right)\right] \boldsymbol{E}_{\mathrm{cl}}=\mathrm{i} \nabla \times \boldsymbol{H}_{\mathrm{cl}}, \quad \frac{\omega}{c} \boldsymbol{H}_{\mathrm{cl}}=-\mathrm{i} \nabla \times \boldsymbol{E}_{\mathrm{cl}},
$$

where $\varepsilon_{\omega}=1+4 \pi \chi_{\omega}$. Quite natural expectation is that in average (over a time period) in electromagnetic wave there is the same quantity of energy in electric as in magnetic field. After neglecting the nonlinear contribution to the energy it means that $\left\langle\varepsilon \boldsymbol{E}(t)^{2}\right\rangle=\left\langle\mu \boldsymbol{H}(t)^{2}\right\rangle$, and thus $\boldsymbol{E}_{\mathrm{cl}}^{2} \propto \boldsymbol{H}_{\mathrm{cl}}^{2}$. Because of the smallness of the $\alpha_{\omega}$ one can write

$$
\frac{\omega}{c} \varepsilon_{\mathrm{NL}} \boldsymbol{E}_{\mathrm{cl}}=\mathrm{i} \nabla \times \boldsymbol{H}_{\mathrm{cl}}, \quad \frac{\omega}{c} \boldsymbol{H}_{\mathrm{cl}}=-\mathrm{i} \nabla \times \boldsymbol{E}_{\mathrm{cl}},
$$

where

$$
\varepsilon_{\mathrm{NL}}=\varepsilon_{\omega}+\tilde{\alpha}_{\omega} \boldsymbol{E}_{\mathrm{cl}}^{2} .
$$

The interaction between photons results as the Kerr type nonlinearity of the medium (22). Such type of nonlinearity is required to obtain (in paraxial and slowly varying envelop approximation) soliton solutions of the Maxwell equations [10]. In 
the view of the above results the soliton can be treated as a bound state (cluster) of many photons. This is the attracting interaction between photons, which can counteract spreading (because of dispersion or diffraction) of the wave packet.

\section{Summary}

The influence of the medium on photon can be described by some scalar potentials. This approach can be easily extended to the case of many interacting photons. Taking the simplest model of such interaction (via delta-potential) in the Hartree approximation one obtains the Kerr type nonlinearity of the medium. This result suggests that optical solitons are nothing else but clusters of interacting photons. In such a cluster the attraction between photons counteracts dispersion or diffraction. This is a similar point of view to that presented in [11] where solitons of magnetization in magnetics were described as clusters of interacting (via delta-potential) magnons.

\section{References}

[1] I. Białynicki-Birula, Acta Phys. Pol. A 86, 97 (1994); e-print http://www.cft.edu.pl/ birula/publ.html .

[2] I. Białynicki-Birula, in: Coherence and Quantum Optics VII, Eds. J.H. Eberly, L. Mandel, E. Wolf, Plenum, New York 1996, p. 313; e-print http://www.cft.edu.pl/ birula/publ.html .

[3] I. Białynicki-Birula, in: Progress in Optics, Vol. 36, Ed. E. Wolf, Elsevier, Amsterdam 1996, p. 245; e-print http://www.cft.edu.pl/ birula/publ.html .

[4] J.E. Sipe, Phys. Rev. A 52, 1875 (1995).

[5] D.I. Blochincev, Foundations of Quantum Mechanics, Nauka, Moskva 1983 (in Russian).

[6] I.N. Meshkov, B.V. Chirikov, Electromagnetic Field, Izd. Nauka, Novosibirsk 1987 (in Russian).

[7] Y. Lai, H.A. Haus, Phys. Rev. A 40, 844 (1989).

[8] Y. Lai, H.A. Haus, Phys. Rev. A 40, 854 (1989).

[9] A.M. Kosevich, A.S. Kovalev, Introduction to Nonlinear Physical Mechanics, Nauk. dumka, Kiev 1989 (in Russian).

[10] N.N. Akhmediev, A. Ankiewicz, Solitons. Nonlinear Pulses and Beams, Chapman and Hall, London 1997.

[11] A.M. Kosevich, V.A. Ivanov, A.S. Kovalev, Nonlinear Waves of Magnetization. Dynamic and Topological Solitons, Nauk. dumka, Kiev 1983 (in Russian). 